\title{
FIXED POINTS OF ELEMENTARY SUBGROUPS OF CHEVALLEY GROUPS ACTING ON TREES
}

\author{
By \\ Makoto FukunAGA
}

\section{Introduction}

Consider the following condition on a group $G$ :

(FA) For any tree $X$ and any action without inversions of $G$ on $X$, the set $X^{G}$ of fixed points is non-empty.

Jean-Pierre Serre has shown that every group with this property has many interesting group theoretical properties (cf.[5]). He has also shown that the special linear group $S L(2, \boldsymbol{Z})$ of degree 2 over the ring $\boldsymbol{Z}$ of rational integers does not satisfy $(F A)$, but $S L(3, \boldsymbol{Z})$ does. In this paper we shall generalize this result to the elementary subgroup $E_{\rho}(\Phi, R)$ (See Section 2 below.) of a Chevalley group of type $\Phi$ over a commutative ring $R$ with an identity under the assumption that $\Phi$ is irreducible of rank $\geqq 2$ and the additive group $R^{+}$of $R$ is finitely generated. For any group $G$, we use $[G, G]$ to denote the commutator subgroup of $G$ generated by all $[x, y]=x y x^{-1} y^{-1}, x, y \in G$.

I would like to express my warm thanks to Professor Eiichi Abe for his valuable advice during the preparation of this paper.

\section{The action of a group on a tree}

We begin with the definition of graphs. A graph $X=(S(X), \operatorname{Ar}(X))$ consists of a non-empty set $S(X)$ and a subset $\operatorname{Ar}(X)$ of $S(X) \times S(X)$ such that $(s, s) \notin A r(X)$ for any $s \in S(X)$ and $\operatorname{Ar}(X)=\operatorname{Ar}(X)^{t}$, where

$$
\operatorname{Ar}(X)^{t}=\left\{\left(s, s^{\prime}\right) \mid\left(s^{\prime}, s\right) \in \operatorname{Ar}(X)\right\} .
$$

Each element of $S(X)$ (resp. $A r(X)$ ) is called a vertex (resp. an edge). We shall sometimes identify a graph $X$ with the set $S(X)$ of vertices and an edge $\left(s, s^{\prime}\right)$ with $\left(s^{\prime}, s\right)$. A series of finitely many edges $\left(s_{0}, s_{1}\right),\left(s_{1}, s_{2}\right), \cdots,\left(s_{n-1}, s_{n}\right)$ is called a path of length $n$ connecting $s_{0}$ and $s_{n}$, and we shall denote it by $\left(s_{0}, s_{1}, \cdots, s_{n}\right)$. In particular, the path $\left(s_{0}, s_{1}, \cdots, s_{n}\right)$ is called geodesic if the vertices $s_{0}, s_{1}, \cdots, s_{n}$ are all distinct. Any path connecting two distinct vertices can be reduced to a geodesic 
path. On the other hand, the path $\left(s_{0}, s_{1}, \cdots, s_{n}\right)$ is said to be a loop when $n \geqq 3$, $s_{0}=s_{n}$ and the path $\left(s_{1}, s_{2}, \cdots, s_{n}\right)$ is geodesic. A graph is defined to be connected if for any two distinct vertices there is a path connecting these two vertices. A connected graph is called a tree when it has no loops. It is easy to see that any two distinct vertices in a tree are connected by one and only one geodesic path.

An automorphism $f$ of a graph $X$ is a set theoretical bijection from $S(X)$ onto itself such that a pair of vertices $\left(s, s^{\prime}\right)$ is in $\operatorname{Ar}(X)$ if and only if $\left(f(s), f\left(s^{\prime}\right)\right)$ is in $\operatorname{Ar}(X)$. The set of all automorphisms of a graph $X$ forms naturally a group $A u t(X)$ under the composition of maps. We say that a group $G$ acts on a graph $X$ when there is a group homomorphism $\sigma$ of $G$ into $A u t(X)$. Then every element $g$ of $G$ induces an automorphism of $X$ defined by

$$
x \longmapsto \sigma(g) x=g x
$$

Now consider an action of a group $G$ on a tree $X=(S(X), A r(X))$. It sometimes happens that there exist $\left(s, s^{\prime}\right) \in A r(X)$ and $g \in G$ such that $\left(g s, g s^{\prime}\right)=\left(s^{\prime}, s\right)$. In this case we say that this action has an inversion. It is known that an action with inversions can be reduced to the case without inversions by the following method of barycentric subdivision. Assume that $\left(s, s^{\prime}\right) \in A r(X)$ and $g \in G$ is an inversion. Take a tree $X^{\prime}=\left(S\left(X^{\prime}\right), A r\left(X^{\prime}\right)\right)$ in place of $X$, where

$$
\begin{aligned}
& S\left(X^{\prime}\right)=S\left(X^{\prime}\right) \cup\left\{s^{\prime \prime}\right\}, s^{\prime \prime} \notin S(X) \text { and } \\
& \operatorname{Ar}\left(X^{\prime}\right)=\left(\operatorname{Ar}(X)-\left\{\left(s, s^{\prime}\right)\right\}\right) \cup\left\{\left(s, s^{\prime \prime}\right),\left(s^{\prime \prime}, s^{\prime}\right)\right\} .
\end{aligned}
$$

The action of $G$ on $X$ can be naturally extended to $X^{\prime}$ by defining $g s^{\prime \prime}=s^{\prime \prime}$ for all $g \in G$. In this paper we assume that no action of a group on a tree has any inversions.

When a group $G$ acts on a graph $X$, we denote

$$
\begin{aligned}
& X^{G}=\{s \in S(X) \mid g s=s \text { for all } g \in G\} \text { and } \\
& X^{g}=\{s \in S(X) \mid g s=s\}, \text { where } g \text { is a fixed element of } G .
\end{aligned}
$$

Proposition 1. Let $X$ be a tree.

(i) If $X^{G}$ is non-empty, then $X^{G}$ is a tree.

(ii) Let $X_{i}(1 \leqq i \leqq n)$ be subsets of $X$. If each $X_{i}$ is a tree and $X_{i} \cap X_{j}$ is nonempty for all pairs $(i, j)$, then $\bigcap_{1 \leq i \leq n} X_{i}$ is non-empty and connected. 
Proof (i) We shall show that $X^{a}$ is connected. For distinct vertices $s, s^{\prime} \in X^{G}$, there is a unique geodesic path $\left(s, s_{1}, \cdots, s_{n}, s^{\prime}\right)$ in $X$. So $\left(g s, g s_{1}, \cdots, g s_{n}, g s^{\prime}\right)=\left(s, g s_{1}\right.$, $\left.\cdots, g s_{n}, s^{\prime}\right)$ is the same path for all $g \in G$. Therefore $s_{1}, s_{2}, \cdots, s_{n} \in X^{G}$.

(ii) Using induction on $n$, it is enough to show (ii) when $n=3$. Choose any $s_{i j} \in X_{i} \cap X_{j}$ for $i, j=1,2,3$. We may assume $s_{12} \notin X_{3}, s_{23} \notin X_{1}$ and $s_{13} \notin X_{2}$, otherwise the proof is completed. So the three vertices $s_{12}, s_{23}$ and $s_{13}$ are all distinct. Since $s_{12}$ and $s_{28}$ (resp. $s_{23}$ and $s_{13}$ ) are connected by a path in $X_{2}$ (resp. in $X_{3}$ ), there is a path connecting $s_{12}$ and $s_{13}$. Reducing the path to be geodesic, we get a geodesic path connecting $s_{12}$ and $s_{13}$ which runs through $X_{2} \cap X_{3}$. But by the uniqueness of geodesic paths, this path is contained in $X_{1}$. So we have $X_{1} \cap X_{2} \cap X_{3} \neq \phi$. The connectedness of $X_{1} \cap X_{2} \cap X_{3}$ is obvious.

q.e.d.

\section{Elementary subgroups of Chevalley groups and some of their properties}

In this section we recall the definition of elementary subgroups of Chevalley groups and give some of their properties. Let $\Phi$ be a (reduced) irreducible root system (cf. [2], Chap. 6), and $V$ be the real Euclidean space spanned by $\Phi$. When we choose a base $\Delta$ of the root system $\Phi$, the set of positive (resp. negative) roots with respect to $\Delta$ is determined and we shall denote it by $\Phi^{+}$(resp. $\Phi^{-}$). Let $\Delta^{\prime}$ be a non-empty subset of $\Delta=\left\{\alpha_{i}\right\}$. Then

$$
\left\{\Sigma n_{i} \alpha_{i} \in \Phi \mid n_{i}=0 \text { if } \alpha_{i} \notin \Delta^{\prime}\right\}
$$

is a (not necessarily irreducible) root system with a base $\Delta^{\prime}$, and we shall denote it by

$$
\left\langle\alpha_{i} \mid \alpha_{i} \in \Delta^{\prime}\right\rangle
$$

Each root $\alpha \in \Phi$ defines a reflection $r_{\alpha}$ of the space $V$, which sends $\alpha$ to $-\alpha$ and leaves pointwise fixed the hyperplane orthogonal to $\alpha$. All reflections determined by the roots of $\Phi$ generate a group $W$ called the Weyl group of $\Phi$.

Each irreducible root system $\Phi$ determines uniquely (up to isomorphism) a finite dimensional simple Lie algebra $g(\Phi)$ over the field of complex numbers. Let $\rho$ be a faithful representation of the Lie algebra $g(\Phi)$ on a finite dimensional vector space over the field of complex numbers, then we can construct the Chevalley-Demazure group scheme $G_{\rho}(\Phi, \quad)$ associated with $\Phi$ and $\rho$ (cf. [1], [3] and [7]). Since $G_{\rho}(\Phi, \quad)$ is a covariant functor from the category of commutative rings to the category of groups, we get a group $G_{\rho}(\Phi, R)$ of the points of $G_{\rho}(\Phi, \quad)$ in a commutative ring $R$ with an identity. In particular, if $R=C$ is the field of complex numbers, $G_{\rho}(\Phi, \boldsymbol{C})$ has the structure of a Lie group. $G_{\rho}(\Phi, \quad)$ is called 
simply connected when the Lie group $G_{\rho}(\Phi, C)$ is simply connected, or equivalently when the set of fundamental weights is a base of the lattice generated by the set of all weights of the representation $\rho$. We shall give an example. Assume that $\Phi$ is of type $A_{l}$ and $G_{\rho}(\Phi, \quad)$ is simply connected. Then $G_{\rho}(\Phi, R)$ is isomorphic to the special linear group $S L(l+1, R)$ of degree $l+1$ over a commutative ring $R$ with an identity. In general, when $\Phi$ is of type $A_{l}, G_{\rho}(\Phi, R)$ is isomorphic to a quotient group of $S L(l+1, R)$ by a central subgroup.

For each root $\alpha \in \Phi$, there is a group isomorphism

$$
t \longmapsto x_{\alpha}(t)
$$

of the additive group $R^{+}$of $R$ onto a subgroup $X_{\alpha}$ of $G_{\rho}(\Phi, R) . \quad X_{\alpha}$ is called the root subgroup corresponding to the root $\alpha$. The elementary subgroup $E_{\rho}(\Phi, R)$ is defined to be the subgroup of $G_{\rho}(\Phi, R)$ generated by all $X_{\alpha}$ for $\alpha \in \Phi$. When $G_{\rho}(\Phi, \quad)$ is simply connected, $E_{\rho}(\Phi, R)$ is equal to $G_{\rho}(\Phi, R)$ if $R$ is a local ring (cf. [1], Proposition 1.6) or $R$ is a Euclidean domain (cf. [7], §8). But, in general, $E_{\rho}(\Phi, R)$ is a proper subgroup of $G_{\rho}(\Phi, R)$. For a base $\Delta$ of $\Phi$, let $U_{\rho}(\Phi, R, \Delta)$ be the subgroup of $E_{\rho}(\Phi, R)$ generated by all $X_{\alpha}$ for $\alpha \in \Phi^{+}$. Then $U_{\rho}(\Phi, R, \Delta)$ is unipotent and hence nilpotent (cf. [7], p. 26).

Now we shall make a list of some relations between generators in the elementary subgroup $E_{\rho}(\Phi, R)$ (cf. [3], [6] and [7]).

(RI) For any $s, t \in R$ and $\alpha \in \Phi$,

$$
x_{\alpha}(s) x_{\alpha}(t)=x_{\alpha}(s+t) .
$$

(RII) Let rank $\Phi=l \geqq 2$. For any $s, t \in R$ and $\alpha, \beta \in \Phi$ such that $\alpha+\beta \neq 0$,

$$
\left[x_{\alpha}(s), x(t)\right]=\Pi x_{i \alpha+j \beta}\left(N_{\alpha, \beta, i, j} s^{i} t^{j}\right)
$$

where the product on the right is taken over all roots of the form $i \alpha+j \beta$, for positive integers $i$ and $j$ arranged in some fixed order, and $N_{\alpha, \beta, i, j}$ are integers depending only on $\alpha, \beta$ and the chosen ordering.

$(R I I I) \quad$ For any $t \in R$ and $\alpha, \beta \in \Phi$

$$
w_{\alpha} x_{\beta}(t) w_{\alpha}^{-1}=x_{r_{\alpha}(\beta)}( \pm t),
$$

where $w_{\alpha}=x_{\alpha}(1) x_{-\alpha}(-1) x_{\alpha}(1)$.

Proposition 2. Let $R$ be a commutative ring with an identity and $\Phi$ be an irreducible root system of rank $\geqq 2$. For each $x_{\alpha}(t) \in E_{\rho}(\Phi, R), \alpha \in \Phi, t \in R$, there exist a positive integer $n$ and $a$ base $\Delta$ of $\Phi$ such that

$$
x_{\alpha}(t) \in U
$$

$$
x_{\alpha}(t)^{n}=x_{\alpha}(n t) \in[U, U] \text {, where } U=U_{\rho}(\Phi, R, \Delta)
$$


Proof. Choosing a suitable base $\Delta$, we may assume that the given root $\alpha$ is positive and not simple. Furthermore we may assume that $\Phi$ is an irreducible root system of rank 2 , that is, $\Phi=A_{2}$, or $B_{2}$, or $G_{2}$. Since the proof of the case $A_{2}$ or $B_{2}$ is easy, we shall prove the most complicated case $\Phi=G_{2}$. Set $\Delta=\left\{\alpha_{1}, \alpha_{2}\right\}$ and $\Phi=\left\{ \pm \alpha_{1}, \pm \alpha_{2}, \pm\left(\alpha_{1}+\alpha_{2}\right), \pm\left(2 \alpha_{1}+\alpha_{2}\right), \pm\left(3 \alpha_{1}+\alpha_{2}\right), \pm\left(3 \alpha_{1}+2 \alpha_{2}\right)\right\}$, then $\alpha=\alpha_{1}+\alpha_{2}$, or $2 \alpha_{1}+\alpha_{2}$, or $3 \alpha_{1}+\alpha_{2}$, or $3 \alpha_{1}+2 \alpha_{2}$. As special relations of $(R I I)$, we have

$$
\begin{aligned}
& {\left[x_{\alpha_{2}}(s), x_{3 \alpha_{2}+\alpha_{2}}(t)\right]=x_{3 \alpha_{1}+2 \alpha_{2}}( \pm s t),} \\
& {\left[x_{2 \alpha_{1}+\alpha_{2}}(s), x_{\alpha_{1}}(t)\right]=x_{3 \alpha_{1}+\alpha_{2}}( \pm 3 s t),} \\
& {\left[x_{\alpha_{1}+\alpha_{2}}(s), x_{\alpha_{1}}(t)\right]=x_{2 \alpha_{1}+\alpha_{2}}( \pm 2 s t) x_{3 \alpha_{1}+\alpha_{2}}\left( \pm 3 s t^{2}\right) x_{3 \alpha_{1}+2 \alpha_{2}}\left( \pm 3 s^{2} t\right) \text { and }} \\
& {\left[x_{\alpha_{1}}(s), x_{\alpha_{2}}(t)\right]=x_{\alpha_{1}+\alpha_{2}}( \pm s t) x_{2 \alpha_{1}+\alpha_{2}}\left( \pm s^{2} t\right) x_{3 \alpha_{1}+\alpha_{2}}\left( \pm s^{3} t\right) x_{3 \alpha_{1}+2 \alpha_{2}}\left( \pm 2 s^{3} t^{2}\right) .}
\end{aligned}
$$

So we can choose $n=1$ (resp. $n=2, n=3, n=6$ ), when $\alpha=3 \alpha_{1}+2 \alpha_{2}$ (resp. $\alpha=2 \alpha_{1}+\alpha_{2}$, $\left.\alpha=3 \alpha_{1}+\alpha_{2}, \alpha=\alpha_{1}+\alpha_{2}\right)$.

q.e.d.

For an irreducible root system $\Phi$ the highest root exists uniquely with respect to some fixed base of $\Phi$ (cf. [2], Chap. 6).

Proposition 3. Let $\Phi$ be an irreducible root system of rank $\geqq 3$ with a base $\Delta=\left\{\alpha_{1}, \alpha_{2}, \cdots, \alpha_{l}\right\}$. Then we can choose $\alpha_{1}$ such that the following are satisfied.

(i) $\Phi^{\prime}=\left\langle\alpha_{2}, \cdots, \alpha_{l}\right\rangle$ is an irreducible root system of rank $l-1$ with a base $\Delta^{\prime}=$ $\left\{\alpha_{2}, \cdots, \alpha_{l}\right\}$.

(ii) Let $\alpha_{0}$ and $\beta_{0}$ be the highest roots of $\Phi$ and $\Phi^{\prime}$ with respect to the base $\Delta$ and $\Delta^{\prime}$ respectively. If $\Phi$ is not of type $C_{l}$ or $F_{4}$ (resp. $\Phi$ is of type $C_{l}$ or $F_{4}$ ), put $\gamma_{1}=\alpha_{0}-\beta_{0}$ (resp. $\left.2 \gamma_{1}=\alpha_{0}-\beta_{0}\right)$. Then $\gamma_{1} \in \Phi^{+}$and $\left\langle\beta_{0}, \gamma_{1}\right\rangle$ is of type $A_{2}$ (resp. of type $B_{2}$ ) with highest root $\alpha_{0}$.

(iii) Put $\gamma_{2}=\gamma_{1}-\alpha_{1}$. Then if $\Phi$ is of type $A_{l}$ or $C_{l}, \gamma_{2}=0$. Otherwise, $\gamma_{2} \in \Phi^{+}$.

(iv) If $\Phi$ is not of type $A_{l}$ or $C_{l}$ or $F_{4}$, then $\left\langle\alpha_{1}, \gamma_{2}\right\rangle$ is of type $A_{2}$ and the highest root is $\gamma_{1}$.

(v) If $\Phi$ is of type $F_{4}$, then $\left\langle\alpha_{1}, \gamma_{2}\right\rangle$ is of type $B_{2}$ and the highest root is $\alpha_{1}+2 \gamma_{2}$.

Proof. The proposition can be proved by the classification of irreducible root systems (cf. [2] Chap. 6). For each system, we give the Dynkin diagram having $\alpha_{1}$ as a terminal node, the type of $\Phi^{\prime}$ and the expressions of $\alpha_{0}, \beta_{0}, \gamma_{1}$ and $\gamma_{2}$ by the base of $\Phi$.

$A_{l}(l \geqq 3)$

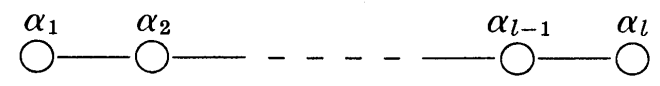


$\Phi^{\prime}$ is of type $A_{l-1}$.

$\alpha_{0}=\alpha_{1}+\alpha_{2}+\cdots \cdots+\alpha_{l}$,

$\beta_{0}=\alpha_{2}+\alpha_{3}+\cdots \cdots+\alpha_{l}$,

$\gamma_{1}=\alpha_{1} \quad$ and $\quad \gamma_{2}=0$.

$B_{l}(l \geqq 3)$

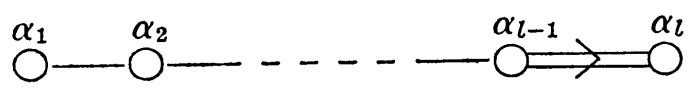

$\Phi^{\prime}$ is of type $B_{l-1}$.

$$
\begin{aligned}
& \alpha_{0}=\alpha_{1}+2\left(\alpha_{2}+\alpha_{3}+\cdots \cdots+\alpha_{l}\right), \\
& \beta_{0}=\alpha_{2}+2\left(\alpha_{3}+\cdots \cdots+\alpha_{l}\right), \\
& \gamma_{1}=\alpha_{1}+\alpha_{2} \quad \text { and } \gamma_{2}=\alpha_{2} .
\end{aligned}
$$

$C_{l}(l \geqq 3)$

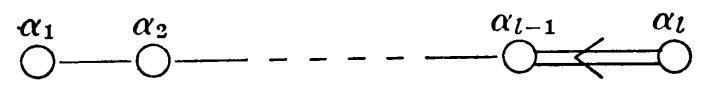

$\Phi^{\prime}$ is of type $C_{l-1}$.

$$
\begin{aligned}
& \alpha_{0}=2\left(\alpha_{1}+\cdots \cdots+\alpha_{l-1}\right)+\alpha_{l}, \\
& \beta_{0}=2\left(\alpha_{2}+\cdots \cdots+\alpha_{l-1}\right)+\alpha_{l}, \\
& \gamma_{1}=\alpha_{1} \quad \text { and } \quad \gamma_{2}=0 .
\end{aligned}
$$

$D_{l}(l \geqq 4)$

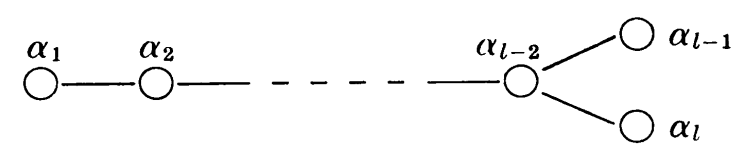

$\Phi^{\prime}$ is of type $A_{3}$ (if $l=4$ ) or $D_{l-1}($ if $l \geqq 5$ ).

$\alpha_{0}=\alpha_{1}+2\left(\alpha_{2}+\cdots \cdots+\alpha_{l-2}\right)+\alpha_{l-1}+\alpha_{l}$,

$\beta_{0}=\alpha_{2}+2\left(\alpha_{3}+\cdots \cdots+\alpha_{l-2}\right)+\alpha_{l-1}+\alpha_{l}$,

$\gamma_{1}=\alpha_{1}+\alpha_{2} \quad$ and $\gamma_{2}=\alpha_{2}$.

$E_{6}$

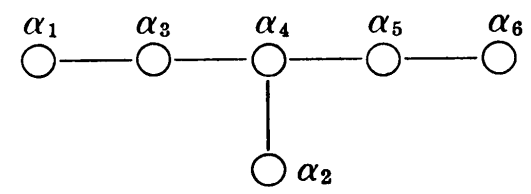

$\Phi^{\prime}$ is of type $D_{4}$.

$\alpha_{0}=\alpha_{1}+2 \alpha_{2}+2 \alpha_{3}+3 \alpha_{4}+2 \alpha_{5}+\alpha_{6}$,

$\beta_{0}=\alpha_{2}+\alpha_{3}+2\left(\alpha_{4}+\alpha_{5}\right)+\alpha_{6}$, 
Fixed points of elementary subgroups of Chevalley groups

$$
\begin{aligned}
& \gamma_{1}=\alpha_{1}+\alpha_{2}+\alpha_{3}+\alpha_{4} \quad \text { and } \\
& \gamma_{2}=\alpha_{2}+\alpha_{3}+\alpha_{4} .
\end{aligned}
$$

$E_{7}$

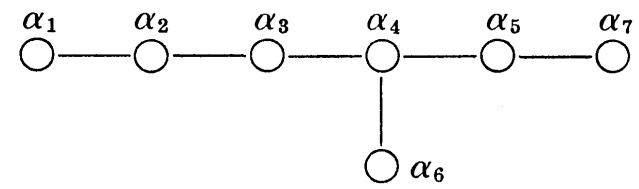

$\Phi^{\prime}$ is of type $E_{6}$.

$\alpha_{0}=\alpha_{1}+2 \alpha_{2}+3 \alpha_{3}+4 \alpha_{4}+3 \alpha_{5}+2 \alpha_{6}+2 \alpha_{7}$,

$\beta_{0}=\alpha_{2}+2 \alpha_{3}+3 \alpha_{4}+2 \alpha_{5}+2 \alpha_{6}+\alpha_{7}$,

$\gamma_{1}=\alpha_{1}+\alpha_{2}+\alpha_{3}+\alpha_{4}+\alpha_{5}+\alpha_{7} \quad$ and

$\gamma_{2}=\alpha_{2}+\alpha_{3}+\alpha_{4}+\alpha_{5}+\alpha_{7}$.

$E_{8}$

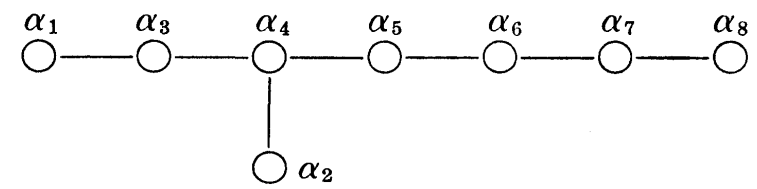

$\Phi^{\prime}$ is of type $D_{7}$.

$\alpha_{0}=2 \alpha_{1}+3 \alpha_{2}+4 \alpha_{3}+6 \alpha_{4}+5 \alpha_{5}+4 \alpha_{6}+3 \alpha_{7}+2 \alpha_{8}$,

$\beta_{0}=\alpha_{2}+\alpha_{3}+2\left(\alpha_{4}+\alpha_{5}+\alpha_{6}+\alpha_{7}\right)+\alpha_{8}$,

$\gamma_{1}=2 \alpha_{1}+2 \alpha_{2}+3 \alpha_{3}+4 \alpha_{4}+3 \alpha_{5}+2 \alpha_{6}+\alpha_{7}+\alpha_{8} \quad$ and

$\gamma_{2}=\alpha_{1}+2 \alpha_{2}+3 \alpha_{3}+4 \alpha_{4}+3 \alpha_{5}+2 \alpha_{6}+\alpha_{7}+\alpha_{8}$.

$F_{4}$

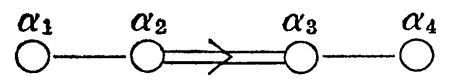

$\Phi^{\prime}$ is of type $C_{3}$.

$\alpha_{0}=2 \alpha_{1}+3 \alpha_{2}+4 \alpha_{3}+2 \alpha_{4}$,

$\beta_{0}=\alpha_{2}+2 \alpha_{3}+2 \alpha_{4}$,

$\gamma_{1}=\alpha_{1}+\alpha_{2}+\alpha_{3} \quad$ and

$\gamma_{2}=\alpha_{2}+\alpha_{3}$.

q.e.d.

Proposition 4. Let $\Phi$ be an irreducible root system of rank $\geqq 2$ and $\Delta$ be any fixed base of $\Phi$. Then the elementary subgroup $E_{\rho}(\Phi, R)$ is generated by $\left\{X_{\alpha} \mid \alpha \in \Psi\right\}$, where

$$
\Psi=\left(\Phi^{+}-\left\{\alpha_{0}\right\}\right) \cup\left\{-\alpha_{0}\right\}
$$


and $\alpha_{0}$ is the highest root of $\Phi$ with respect to $\Delta$.

Proof. First we shall prove this in case the rank of $\Phi$ is 2 , that is, $\Phi$ is of type $A_{2}$, or $B_{2}$, or $G_{2}$. Then we shall treat the case when the rank of $\Phi$ is greater than 2. Let $G[\Psi]$ be the subgroup of $E_{\rho}(\Phi, R)$ generated by $\left\{X_{\alpha} \mid \alpha \in \Psi\right\}$. We have to show that $G[\Psi]$ is equal to $E_{\rho}(\Phi, R)$.

Supposing first that we are in case $A_{2}$, or $B_{2}$, or $G_{2}$, put $\Delta=\{\alpha, \beta\}$ such that $\alpha$ is a short root and $\beta$ is a long root if $\Phi$ is of type $B_{2}$ or $G_{2}$. Then $\alpha_{0}=\alpha+\beta$ (resp. $\alpha_{0}=2 \alpha+\beta, \alpha_{0}=3 \alpha+2 \beta$ ) when $\Phi$ is $A_{2}$ (resp. $B_{2}, G_{2}$ ). We claim that there exists a base $\Delta^{\prime}=\left\{\alpha^{\prime}, \beta^{\prime}\right\}$ such that root subgroups corresponding to the roots $\pm \alpha^{\prime}$ and $\pm \beta^{\prime}$ are contained in $G[\Phi]$. Since we have a relation (cf. [7], §3 and §4)

$$
\left[x_{\alpha}(s), x_{\beta}(t)\right]=x_{\alpha+\beta}( \pm s t)
$$

(resp. $\left.\left[x_{\alpha}(s), x_{\beta}(t)\right]=x_{\alpha+\beta}( \pm s t) x_{2 \alpha+\beta}\left( \pm s^{2} t\right),\left[x_{\beta}(s), x_{3 \alpha+\beta}(t)\right]=x_{3 \alpha+2 \beta}( \pm s t)\right)$

when $\Phi$ is $A_{2}$ (resp. $\left.B_{2}, G_{2}\right), X_{\alpha_{0}} \subseteq G[\Psi]$ and hence $w_{\alpha_{0}}=x_{\alpha_{0}}(1) x_{-\alpha_{0}}(-1) x_{\alpha_{0}}(1) \in G[\Psi]$. By $(R I I I)$, every root subgroup corresponding to the root conjugate to a positive root by $w_{\alpha_{0}}$ is in $G[\Psi]$ (cf. [4], $\S 4$ ). So we can choose $\Delta^{\prime}=\Delta=\{\alpha, \beta\}$ (resp. $\Delta^{\prime}=$ $\left\{\alpha,-(\beta+2 \alpha) \Delta^{\prime}=\{-(\beta+\alpha), \beta\}\right)$ if $\Phi$ is $A_{2}$ (resp. $B_{2}, G_{2}$ ), and the subgroup generated by $\left\{w_{r} \mid \gamma \in \Delta^{\prime}\right\}$ is contained in $G[\Psi]$. Since the Weyl group $W$ of $\Phi$ is generated by the reflections corresponding to the roots of $\Delta^{\prime}$ (cf. [2], Chap. 6, §1, Th. 2), every root subgroup corresponding to the root conjugate to a root of $\Delta^{\prime}$ under $W$ is in $G[\Psi]$. On the other hand, for any two roots of the same length, there is an element of $W$ which maps one to the other (cf. [2], Chap. 6, §1, Prop. 11). Therefore every root subgroup is in $G[\Psi]$, and this completes the proof of this case.

Supposing next that we are in case the rank of $\Phi \geqq 3$, we proceed by induction on the rank and use the notation of Proposition 3. $\left\langle\beta_{0}, \gamma_{1}\right\rangle$ is an irreducible root system of rank 2 with highest root $\alpha_{0}$. By hypothesis, $X_{r} \subseteq G[\Phi]$, where $\gamma$ is any positive root in $\left\langle\beta_{0}, \gamma_{1}\right\rangle$ or $\gamma=-\alpha_{0}$. Then by the cases of rank $2, X_{-\beta_{0}}, X_{-r_{1}} \leqq G[\Phi]$. Since $\beta_{0}$ is the highest root of $\left\langle\alpha_{2}, \cdots, \alpha_{l}\right\rangle, X_{-\alpha_{i}} \leqq G[\Phi](2 \leqq i \leqq l)$ by induction. It remains only to show that $X_{-\alpha_{1}} \subseteq G[\Phi]$. If $\Phi=A_{l}$ or $C_{l}, \gamma_{1}=\alpha_{1}$, hence we have $X_{-\alpha_{1}} \subseteq G[\Phi]$. If $\Phi$ is not $A_{l}$ or $C_{l}$ or $F_{4}$, then $\left\langle\alpha_{1}, \gamma_{2}\right\rangle$ is an irreducible root system of rank 2 with highest root $\gamma_{1}$. By an argument similar to the above, we have $X_{-\alpha_{1}} \subseteq G[\Phi]$. Finally if $\Phi$ is of type $F_{4},\left\langle-\gamma_{1}, \alpha_{1}+2 \gamma_{2}\right\rangle$ is of type $B_{2}$ with highest root $-\alpha_{1}$. Hence we have $X_{-\alpha_{1}} \subseteq G[\Psi]$.

q.e.d.

\section{Main result}

In this section we shall prove the following theorem: 
THEOREM. Let $\Phi$ be an irreducible root system of rank $\geqq 2, R$ be a commutative ring with an identity such that the additive group $R^{+}$of $R$ is finitely generated and $\rho$ be any faithful representation of the Lie algebra $\mathrm{g}(\Phi)$. Then the elementary subgroup $E_{\rho}(\Phi, R)$ has the property $(F A)$.

To prove this theorem we need the following result due to Jean-Pierre Serre (cf. [5], Proposition 2 and its corollaries).

Proposition 5. Let $G$ be a finitely generated nilpotent group. Assume that $G$ acts without inversions on a tree $X$.

(i) Let $\left\{g_{i}\right\}$ be a finite set of generators of $G$. If $X^{g_{i}}$ is non-empty for all $i$, then $X^{G}$ is non-empty.

(ii) Let $g$ be an element of $G$. If $g^{n}$ is in $[G, G]$ for some positive integer $n$, then $X^{g}$ is non-empty.

PROPOSITION 6. Assume that the elementary subgroup $E_{\rho}(\Phi, R)$ acts without inversions on a tree $X$, where $\rho, \Phi$ and $R$ are as in the theorem. Let $U=U_{\rho}(\Phi, R, \Delta)$ be as in Section 2. Then $X^{U}$ is non-empty.

Proof. Let $\Delta$ be any fixed base of $\Phi$. Since $U=U_{\rho}(\Phi, R, \Delta)$ is finitely generated and nilpotent, we can apply (i) of Proposition 5 to the group $U$. It is enough to prove that for each generator $g=x_{\alpha}(t), \alpha \in \Phi^{+}, t \in R$, of $U, X^{g}$ is nonempty. On the other hand, by Proposition 2, for any root $\alpha \in \Phi$ and any element $t \in R$ there exist a base $\Delta^{\prime}$ of $\Phi$ and a positive integer $n$ such that $x_{\alpha}(t) \in U^{\prime}$ and $x_{\alpha}(t)^{n}=x_{\alpha}(n t) \in\left[U^{\prime}, U^{\prime}\right]$, where $U^{\prime}=U_{\rho}\left(\Phi, R, \Delta^{\prime}\right)$. Applying (ii) of Proposition 5 to the group $U^{\prime}$ and an element $g=x_{\alpha}(t) \in U^{\prime}$, we have $X^{g} \neq \phi$.

q.e.d.

Proof of the Theorem. Given an action of $E_{\rho}(\Phi, R)$ on a tree $X$, let $\left\{r_{i} \in R \mid i\right.$ $=1, \cdots, n\}$ be a finite set of generators of $R^{+}$. For each $\alpha \in \Phi$ and $r_{i} \in R$, put

$$
g_{i, \alpha}=x_{\alpha}\left(r_{i}\right), \quad X_{i, \alpha}=X^{g_{i, \alpha}} .
$$

First we claim that $X_{i, \alpha} \cap X_{j, \beta}$ is non-empty for any $\alpha, \beta \in \Psi$ and integers $i, j$ ( $1 \leqq i$, $j \leqq n$ ), where $\Psi$ is as in Proposition 4. We may assume $\alpha \neq \beta$. Since $\alpha+\beta$ is non-zero, there is a base $\Delta^{\prime}$ of $\Phi$ such that $\alpha$ and $\beta$ are positive roots with respect to $\Delta^{\prime}$. Take $U^{\prime}=U_{\rho}\left(\Phi, R, \Delta^{\prime}\right)$, then $X^{U^{\prime}}$ is non-empty by Proposition 6. On the other hand, since $g_{i, \alpha}, g_{j, \beta} \in U^{\prime}$, we have $X_{i, \alpha} \cap X_{j, \beta} \supseteqq X^{U^{\prime}}$. Thus $X_{i, \alpha} \cap X_{j, \beta}$ is nonempty. Hence we have, by Proposition 4,

$$
X^{E_{\rho}(\Phi, R)}=\bigcap_{\substack{1 \leq i \leq n \\ \alpha \in \mathbb{W}}} X_{i, \alpha}
$$

and this is non-empty by (Proposition 1).

q.e.d. 


\section{References}

[1] Abe, E., Chevalley groups over local rings, Tohoku Math. J. 21, (1969) 474-494.

[2] Bourbaki, N., Groupes et algèbres de Lie, Chap. IV, V, et VI, Hermann, Paris, 1968.

[3] Chevalley, C., Certains schèmas de groupes semi-simples, Sèm. Bourbaki, Exp. 219 (1960-61).

[4] Hurley, J., Normality in elementary subgroups of Chevalley groups over rings, Canadian J. Math. 28, (1976) 420-428.

[5] Serre, J. P., Amalgames et points fixes, Proc. Second International Conference, Theory of Groups, Canberra, (1973) 633-640.

[6] Stein, M.R., Generators, Relations and Coverings of Chevalley Groups over commutative rings, Amer. J. Math. 93, (1971) 965-1004.

[ 7 ] Steinberg, R., Lectures on Chevalley groups, Yale University, 1968.

[8] Tits, J., Sur le groupe des automorphismes d'un arbre, Essays on topology and related topics, Mémoires dédiés à Georges de Rham, (1970) 188-211.

Institute of Mathematics

University of Tsukuba

Sakuramura, Ibaraki,

305 Japan 